EPJ Web of Conferences 81, 05028 (2014)

DOI: 10.1051 epjconf/ 20148105028

(C) Owned by the authors, published by EDP Sciences, 2014

\title{
The contribution of multi-channel pion-pion scattering to the final states of $\Upsilon$-meson family decays
}

\author{
Yu.S. Surovtsev ${ }^{1, a}$, P. Bydžovský ${ }^{2}$, T. Gutsche ${ }^{3}$, R. Kamiński $^{4}$, V.E. Lyubovitskij ${ }^{3,5,6}$, and M. Nagy ${ }^{7}$ \\ ${ }^{1}$ Bogoliubov Lab of Theoretical Physics, JINR, 141980 Dubna, Russia \\ ${ }^{2}$ Nuclear Physics Institute, AS CR, 25068 Řež/Prague, Czech Republic \\ ${ }^{3}$ Institut für Theoretische Physik, Universität Tübingen, D-72076 Tübingen, Germany \\ ${ }^{4}$ Institute of Nuclear Physics, PAN, Krakow 31342, Poland \\ ${ }^{5}$ Department of Physics, Tomsk State University, 634050 Tomsk, Russia \\ ${ }^{6}$ Mathematical Physics Department, Tomsk Polytechnic University, Lenin ave. 30, 634050 Tomsk, Russia \\ ${ }^{7}$ Institute of Physics, SAS, Bratislava 84511, Slovak Republic
}

\begin{abstract}
The effect of isoscalar $S$-wave multi-channel pion-pion scattering ( $\pi \pi \rightarrow$ $\pi \pi, K \bar{K}, \eta \eta)$ is considered in the analysis of data on decays $-\Upsilon(2 S) \rightarrow \Upsilon(1 S) \pi \pi$, $\Upsilon(3 S) \rightarrow \Upsilon(1 S) \pi \pi$ and $\Upsilon(3 S) \rightarrow \Upsilon(2 S) \pi \pi$.
\end{abstract}

\section{Introduction}

The analysis which aims at studying the scalar mesons [1] is performed jointly considering the isoscalar S-wave processes $\pi \pi \rightarrow \pi \pi, K \bar{K}, \eta \eta$, described in our model-independent approach, and decays $J / \psi \rightarrow \phi(\pi \pi, K \bar{K}), \psi(2 S) \rightarrow J / \psi(\pi \pi)$ and $\Upsilon(2 S) \rightarrow \Upsilon(1 S) \pi \pi$ [2] with adding here data on $\Upsilon(3 S) \rightarrow \Upsilon(1 S) \pi \pi$ and $\Upsilon(3 S) \rightarrow \Upsilon(2 S) \pi \pi$ [3]. A distinction of the $\Upsilon(3 S)$ decays consists in the fact that in this case a phase space cuts off, as if, possible contributions which might interfere destructively with the $\pi \pi$-scattering contribution giving a characteristic 2-humped shape of the di-pion mass spectrum in $\Upsilon(3 S) \rightarrow \Upsilon(1 S) \pi \pi$. A number of mechanisms were proposed to explain this effect, e.g. [4], however, some of them under doubtful assumptions. Here we give a new and natural explanation of this effect allowing for our previous conclusions on the wide resonances $[2,5]$.

\section{The effect of multi-channel $\pi \pi$ scattering in decays of the $\psi$ - and $\Upsilon$-meson families}

Considering multi-channel $\pi \pi$ scattering, we shall deal with the 3 -channel case $(\pi \pi \rightarrow \pi \pi, K \bar{K}, \eta \eta)$ because it was shown [5] that this is a minimal number of coupled channels needed for obtaining correct values of $f_{0}$-resonance parameters. The 3-channel $S$ matrix is determined on the 8 -sheeted Riemann surface. The matrix elements $S_{i j}$, where $i, j=1,2,3$ denote channels, have the right-hand cuts along the real $s$-axis ( $s$ is the invariant total energy squared), starting with the channel thresholds $s_{i}$, and the left-hand cuts related to the crossed channels. Allowance for the Riemann surface structure is performed taking the uniformizing variable $w$ [2] where we have neglected the $\pi \pi$-threshold branch point and allowed for the $K \bar{K}$ - and $\eta \eta$-threshold branch points and left-hand branch point at $s=0$.

\footnotetext{
a e-mail: surovcev@theor.jinr.ru
} 
Resonance representations on the Riemann surface are obtained using formulas from [2], expressing analytic continuations of the $S$-matrix elements to all sheets in terms of those on the physical (I) sheet that have only the resonance zeros. In the 3-channel case, there are 7 types of resonances corresponding to 7 possible situations when there are resonance zeros on sheet I only in $S_{11}-(\mathbf{a})$; $S_{22}-(\mathbf{b}) ; \quad S_{33}-(\mathbf{c}) ; \quad S_{11}$ and $S_{22}-(\mathbf{d}) ; \quad S_{22}$ and $S_{33}-(\mathbf{e}) ; \quad S_{11}$ and $S_{33}-(\mathbf{f}) ; \quad S_{11}, S_{22}$ and $S_{33}-(\mathbf{g})$. Resonances of these types are represented by the pair of complex-conjugate clusters (of poles and zeros on the Riemann surface). The resonance pole clusters of all types in $S_{11}(w)$ on the $w$ plane can be found in [2]. The $S$-matrix elements are taken as $S=S_{B} S_{r e s}$ where $S_{\text {res }}(w)$ includes the model-independent contribution of the resonances, given by the pole clusters. The possible remaining small (model-dependent) contribution of resonances and influence of the channels, not allowed for explicitly in the $w$, are included in $S_{B}$. For the $\pi \pi$ scattering, $S_{B}(\pi \pi)=d_{B}\left(-q_{1}, q_{2}, q_{3}\right) / d_{B}\left(q_{1}, q_{2}, q_{3}\right)$ (analogously for the coupled processes) where

$$
\begin{aligned}
& d_{B}\left(q_{1}, q_{2}, q_{3}\right)=\exp \left\{-i\left[\sum_{n=1}^{3} q_{n}\left(\alpha_{n}+i \beta_{n}\right)\right]\right\} \\
& \alpha_{n}=a_{n 1}+a_{n \sigma}\left(s-s_{\sigma}\right) / s_{\sigma} \theta\left(s-s_{\sigma}\right)+a_{n v}\left(s-s_{v}\right) / s_{v} \theta\left(s-s_{v}\right), \\
& \beta_{n}=b_{n 1}+b_{n \sigma}\left(s-s_{\sigma}\right) / s_{\sigma} \theta\left(s-s_{\sigma}\right)+b_{n v}\left(s-s_{v}\right) / s_{v} \theta\left(s-s_{v}\right)
\end{aligned}
$$

where $q_{n}=\sqrt{s / s_{n}-1}$ and $s_{\sigma}$ is the $\sigma \sigma$ threshold, $s_{v}$ the combined threshold of $\eta \eta^{\prime}, \rho \rho$ and $\omega \omega$ channels. The resonance zeros $w_{r}$ and the background parameters were fixed by fitting to data on $\pi \pi \rightarrow \pi \pi, K \bar{K}, \eta \eta$ with adding the data on $J / \psi \rightarrow \phi(\pi \pi, K \bar{K}), \psi(2 S) \rightarrow J / \psi(\pi \pi), \Upsilon(2 S) \rightarrow \Upsilon(1 S) \pi \pi$ from the Crystal Ball, DM2, Mark II, Mark III, and BES II, Argus, CLEO, CUSB Collaborations (the references, fitting to used data, and the arrangement of resonance poles on the $\sqrt{s}$-plane are in [2]). We have found the following more preferable scenario: the $f_{0}(500)$ is described by the cluster of type (a); the $f_{0}(1370), f_{0}(1500)$ and $f_{0}(1710)$, type $(\mathbf{c})$; and $f_{0}^{\prime}(1500)$, type $(\mathbf{g})$; the $f_{0}(980)$ is represented only by the pole on sheet II and shifted pole on sheet III. The obtained background parameters are: $a_{11}=0.0, a_{1 \sigma}=0.0199, a_{1 v}=0.0, b_{11}=b_{1 \sigma}=0.0, b_{1 v}=0.0338, a_{21}=-2.4649, a_{2 \sigma}=-2.3222$, $a_{2 v}=-6.611, b_{21}=b_{2 \sigma}=0.0, b_{2 v}=7.073, b_{31}=0.6421, b_{3 \sigma}=0.4851, b_{3 v}=0 ; s_{\sigma}=1.6338 \mathrm{GeV}^{2}$, $s_{v}=2.0857 \mathrm{GeV}^{2}$. The very simple description of the $\pi \pi$-scattering background confirms well our assumption $S=S_{B} S_{\text {res }}$ and says that the representation of multi-channel resonances by the pole clusters on the $w$ plane is a good and quite sufficient approach.

The di-meson mass distributions in $J / \psi \rightarrow \phi(\pi \pi, K \bar{K})$ and $V^{\prime} \rightarrow V \pi \pi(V=\psi, \Upsilon)$ are calculated using the formalism from [6]. The decay amplitudes are related to scattering ones $T_{i j}$ as follows:

$$
\begin{aligned}
& F(J / \psi \rightarrow \phi \pi \pi)=\sqrt{2 / 3}\left[c_{1}(s) T_{11}+c_{2}(s) T_{21}\right], \\
& F(J / \psi \rightarrow \phi K \bar{K})=\sqrt{1 / 2}\left[c_{1}(s) T_{12}+c_{2}(s) T_{22}\right], \\
& F(V(2 S) \rightarrow V(1 S) \pi \pi(V=\psi, \Upsilon))=\left[\left(d_{1}, e_{1}\right) T_{11}+\left(d_{2}, e_{2}\right) T_{21}\right], \\
& F(\Upsilon(3 S) \rightarrow \Upsilon(1 S, 2 S) \pi \pi)=\left[\left(f_{1}, g_{1}\right) T_{11}+\left(f_{2}, g_{2}\right) T_{21}\right]
\end{aligned}
$$

where $c_{1}=\gamma_{10}+\gamma_{11} s, c_{2}=\alpha_{2} /\left(s-\beta_{2}\right)+\gamma_{20}+\gamma_{21} s,\left(d_{i}, e_{i}\right)=\left(\delta_{i 0}, \rho_{i 0}\right)+\left(\delta_{i 1}, \rho_{i 1}\right) s$ and $\left(f_{i}, g_{i}\right)=$ $\left(\omega_{i 0}, \tau_{i 0}\right)+\left(\omega_{i 1}, \tau_{i 1}\right) s$ are functions of couplings of the $J / \psi, \psi(2 S), \Upsilon(2 S)$ and $\Upsilon(3 S)$ to a channel $i$. The pole term in $c_{2}$ is an approximation of possible $\phi K$ states, not forbidden by the OZI rules. The expression

$$
N|F|^{2} \sqrt{\left(s-s_{i}\right)\left(m_{\psi}^{2}-\left(\sqrt{s}-m_{\phi}\right)^{2}\right)\left(m_{\psi}^{2}-\left(\sqrt{s}+m_{\phi}\right)^{2}\right)}
$$

for $J / \psi \rightarrow \phi(\pi \pi, K \bar{K})$ and the analogous relations for $V(2 S) \rightarrow V(1 S) \pi \pi(V=\psi, \Upsilon)$ and $\Upsilon(3 S) \rightarrow \Upsilon(1 S, 2 S) \pi \pi$ give the di-meson mass distributions. $\mathrm{N}$ (normalization to experiment) is 0.7512 for Mark III, 0.3705 for DM2, 5.699 for BES II, 1.015 for Mark II, 0.98 for Crystal Ball(80), 4.3439 for Argus, 2.1776 for CLEO, 1.2011 for CUSB, 0.0788 for Crystal 
Ball(85), and, finally, for CLEO(94): 0.5096 and 0.2235 for $\Upsilon(3 S) \rightarrow \Upsilon(1 S)\left(\pi^{+} \pi^{-}\right.$and $\left.\pi^{0} \pi^{0}\right)$, 11.6092 and 5.7875 for $\Upsilon(3 S) \rightarrow \Upsilon(2 S)\left(\pi^{+} \pi^{-}\right.$and $\left.\pi^{0} \pi^{0}\right)$, respectively. We have obtained: $\left(\alpha_{2}, \beta_{2}\right)=(0.0843,0.0385),\left(\gamma_{10}, \gamma_{11}, \gamma_{20}, \gamma_{21}\right)=(1.1826,1.2798,-1.9393,-0.9808)$, $\left(\delta_{10}, \delta_{11}, \delta_{20}, \delta_{21}\right)=(-0.1270,16.621,5.983,-57.653),\left(\rho_{10}, \rho_{11}, \rho_{20}, \rho_{21}\right)=(0.4050,47.0963$, $1.3352,-21.4343),\left(\omega_{10}, \omega_{11}, \omega_{20}, \omega_{21}\right)=(1.1619,-2.915,0.7841,1.0179),\left(\tau_{10}, \tau_{11}, \tau_{20}, \tau_{21}\right)=$ $(7.2842,-2.5599,0.0,0.0)$. A satisfactory combined description of all considered processes is obtained with the total $\chi^{2} / \mathrm{ndf}=596.706 /(527-78) \approx 1.33$. Results of our fitting to the data on decays $\Upsilon(3 S) \rightarrow \Upsilon(1 S) \pi \pi$ and $\Upsilon(3 S) \rightarrow \Upsilon(2 S) \pi \pi$ are shown in figure 1 .
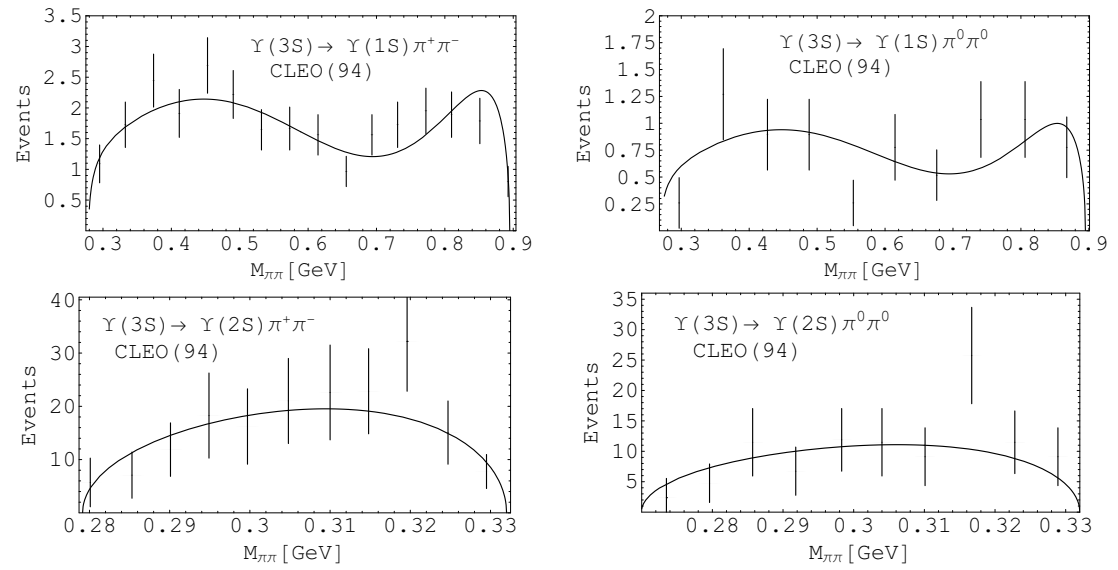

Figure 1. The decays $\Upsilon(3 S) \rightarrow \Upsilon(1 S) \pi \pi$ and $\Upsilon(3 S) \rightarrow \Upsilon(2 S) \pi \pi$; data from the CLEO(94) Collaboration [3].

\section{Conclusions}

It was shown that in the final states of the $\Upsilon$-meson family decays (except the $\pi \pi$ scattering) the contributions of the coupled processes, e.g., $K \bar{K} \rightarrow \pi \pi$, are important even if these processes are energetically forbidden. This is in accordance with our previous conclusions on wide resonances $[2,5]$. E.g., on the basis of that consideration the new and natural mechanism of the destructive interference in the decay $\Upsilon(3 S) \rightarrow \Upsilon(1 S) \pi \pi$ is indicated, which provides the two-humped shape of the di-pion mass distribution. Results of this analysis confirm all of our earlier conclusions on the scalar mesons [2].

This work was supported in part by the Heisenberg-Landau Program, the Votruba-Blokhintsev Program for Cooperation of Czech Republic with JINR, the Grant Agency of the Czech Republic (grant No. P203/12/2126), the Bogoliubov-Infeld Program for Cooperation of Poland with JINR, the DFG under Contract No. LY 114/2-1, the Tomsk State University Competitiveness Improvement Program, and by the Polish National Science Center (NCN) grant DEC-2013/09/B/ST2/04382.

\section{References}

[1] J. Beringer et al. [PDG], Phys. Rev. D 86, 010001 (2012).

[2] Yu.S. Surovtsev et al., Phys. Rev. D 89, 036010 (2014).

[3] F.Butler et al. (CLEO(94)), Phys. Rev. D 49, 40 (1994).

[4] H.J. Lipkin and S.F. Tuan, Phys. Lett. B 206, 349 (1988); P. Moxhay, Phys. Rev. D 39, 3497 (1989); T. Komada et al., AIP Conf. Proc. 619, 499 (2002).

[5] Yu.S. Surovtsev et al., J. Phys. G Nucl. Part. Phys. 41, 025006 (2014).

[6] D. Morgan and M.R. Pennington, Phys. Rev. D 48, 1185, 5422 (1993). 